\title{
SHIELDED-TIP/CANTILEVER PROCESS AND INTERFACE FOR MULTIFUNCTIONAL SCANNING PROBE MICROSCOPY
}

\author{
Toralf Bork*, Vivek Agrawal*, Björn Rosner, Patrick Gustafson, and Daniel van der Weide \\ Department of Electrical \& Computer Engineering \\ University of Wisconsin \\ Madison, WI 53706 \\ "Department of Electrical \& Computer Engineering \\ University of Delaware \\ Newark, DE 19716
}

\begin{abstract}
Along with measuring a sample's topography, coaxial scanning probe microscope (SPM) tips enable electric field imaging [1-3] with completely shielded tips, and both optical [4-6] and microwave [7] detection when diode junctions are formed at the tips. Integrating such tips with appropriate cantilevers and interfaces to external instruments will further promote their applications in scanning capacitance and thermal microscopy, and in electrochemistry and biology. We have batch-fabricated both coaxial and junction tips integrated with matching cantilevers on silicon. We describe the process, show initial results from junction tips as photodetectors, and discuss an electrical and mechanical interface from the probe body to external instrumentation.
\end{abstract}

\section{INTRODUCTION}

Near-field scanning optical microscopy [8-11] (NSOM) is a techniquc by which visible or near-visible light (free-space wavelength generally less than one micrometer) is confined within a sub-wavelength aperture (or is scattered by a sub-wavelength tip [12]) to excite or detect evanescent waves on a sample. A propagating wave incident on a small aperture or scattering center creates evanescent fields. By reciprocity, the aperture, when in proximity to such fields, should radiate propagating waves. To circumvent the far-field resolution Rayleigh limit of $\lambda / 2$ requires that we scan the aperture over the sample. NSOM in the visible has been applied to a growing variety of imaging applications, from localized spectroscopy on semiconductor quantum wells [13] to observing fast thermal transport through conductors on integrated circuits [14] to observing ion transport through cell membranes [15].

Obtaining sub-Rayleigh resolution by trading the multiplex advantage of conventional optics for serial image acquisition is, however, a general principle, and need not be restricted to visible radiation. Ash and Nichols demonstrated this in 1972 using $3 \mathrm{~cm}$ wavelength microwaves confined to a sub-wavelength aperture to achieve $1 / 60$ wavelength-relative resolution [16]. Since resolution is no longer limited by the wavelength of illumination, it is now possible to build microscopes for frequencies below the visible regime. There are myriad electromagnetic phenomena with characteristic frequencies below the visible but above the radio. Examples include integrated circuit (IC) electromagnetic fields (between $100 \mathrm{MHz}$ and $300 \mathrm{GHz}$ ), biological membrane absorption in the far-infrared (FIR) range of $100 \mathrm{GHz}$ to $30 \mathrm{THz}(10 \mu \mathrm{m})$, molecular rotational or vibrational absorption (also primarily in FIR), and conductive or dielectric properties of materials. Just as far field optics enable traditional spectroscopy, which measures the collective response of large numbers of elements (such as molecular electric dipoles), near field techniques can now enable localized spectroscopy, though momentum is no longer conserved, changing the absorption and excitation spectra, especially in the visible and near-infrared.

\section{COAXIAL PROBES FOR NEAR-FIELD MICROSCOPY IN THE SUB-VISIBLE}

One approach to microscopy at wavelengths longer than visible light is to use near field antennas-primarily coaxial probes for electric field sensitivity. Seminal work demonstrating subwavelength field resolution with a coaxial tip was done by Fee, et al. [17], and using open-ended coaxial waveguide for measuring bulk microwave dielectric properties of materials is a common technique [18].

The advantage of using coaxial waveguide for near field imaging in the sub-visible regime is that the shield greatly reduces interference from stray radiation while enabling a non-cutoff transverse electromagnetic (TEM) mode of wave propagation up to or away from the tip. Furthermore, the tip itself can be sharpened to increase the "lightning rod" effect of field concentration, improving resolution. Finally, if the tip is sufficiently sharp, it can also function independently as an SPM topography probe. This multifunctional ability is particularly important in submicrometer field resolution, since the tip-sample distance should be stabilized independent of the field measurement.

Near field antenna probes can be used in different modes, depending on the image to be acquired. These include reflection, transmission, excitation and detection. Reflection and transmission rely on external instruments (lasers or signal generators) for both generating and detecting spectroscopic energy, while excitation might stimulate a photoconductive response, like a change in current through a semiconductor or cell membrane. Detection measures an excited or active sample, such as a waveguide or a circuit; for this mode, integrated photodetectors have been developed [4-6].

In reflection mode, the most widely used by those interested in high spatial resolution of microwave material properties [19-22], the same coaxial probe tip conducts energy both to and from the sample. Although it is necessary to have a closed loop SPM platform to acquire sub-micrometer topography and field images, open loop scanning with sharpened coaxial tips has produced quantitative spatially resolved material measurements such as surface resistivity, especially when the tips are coupled to resonant cavities to enhance the signal.

Although resonant measurements offer high sensitivity, they are also limited in frequency coverage, stimulating the 
development of broadband probes for localized spectroscopy. With sufficiently sensitive microwave instrumentation, such as a vector network analyzer or bridge detector, broadband probes would offer the widest range of applications. Broadband capability is equally important for the transmission mode of sub-visible microscopy [23], in which one probe acts as a source while another on the opposite side of a transmitting sample acts as a detector.

To achieve the highest spatial resolution, both in topography and field, we have developed multifunctional SPM tips with integral near field antennas, both magnetic field loop probes [24] and silicon coaxial probe tips [1]. When integrated with broadband transmission lines and SFM cantilevers as shown here, these tips can provide field and topographic resolution commensurate with their tip radii, which can be $\sim 10 \mathrm{~nm}$.

The final two modes, excitation and detection, can be realized either with external microwave instruments or, in the case of detection, with a sensitive element integrated directly into the antenna. By modifying a commercial SFM tip/cantilever to have coaxial shielding, and connecting this probe to a $50 \mathrm{GHz}$ sampling oscilloscope, we have measured picosecond waveforms and with both submicrometer field and topographic resolution on an IC [2], and have employed a microwave detector diode directly at the coaxial center conductor [7]. With this structure, using a lock-in amplifier we were able to detect the magnitude and phase of a 2 $\mathrm{GHz}$ signal on an interdigitated capacitor sample, as shown in Figure 5. This type of integrated detector has also been used for visible NSOM [4], and it considerably simplifies the instrumentation needed for localized microwave detection.

\section{PROCESS AND INTERFACE DETAILS}

The process for both coaxial and junction tips is similar, but the interface from the cantilever to the outside world is different: for low-loss microwave propagation from coaxial tips we suspend a controlled-impedance coplanar waveguide (CPW) transmission line on a dielectric membrane using $\mathrm{XeF}_{2}$ to undercut the structure [25] as shown in Figs. 1 and 2. The common steps of the process for both types of tips, using a $350 \mu \mathrm{m},(100) 3$ " double-side polished, $\mathrm{n}$ doped, $1-20$ Ohm-cm, TTV $\sim 2 \mu \mathrm{m}$ wafer, are:

1. LPCVD Nitride deposition at $850^{\circ} \mathrm{C}$ for $30 \mathrm{~min}$ as a mask for KOH etching

2. FSC spinning to protect the front side of wafer

3. Lithography: Pattern the nitride as a mask for backside $\mathrm{KOH}$ etch to form the chip body

4. Nitride etch using $\mathrm{CF}_{4}$ plasma process

5. Body formation. $30 \% \mathrm{KOH}$ at $80^{\circ} \mathrm{C}$ to etch to $-270 \mu \mathrm{m}$ deep

6. Removal of all nitride on front side using $\mathrm{CF}_{4}$

7. 1.2 $\mu \mathrm{m}$ PECVD oxide deposition to mask for cantilever and tip

formation

8. Lithography: Pattern the oxide, which acts as a mask for the silicon etch to form the cantilevers

9. Oxide etch to form the cantilever using BHF $6: 1$

10. Cantilever formation: Using anisotropic plasma process to form Si cantilevers

11. Lithography: Pattern the oxide as a mask for the silicon etch to form the tips

12. Oxide etch to form the cantilever using BHF 6:1

13. Cantilever formation: Using anisotropic plasma process to form Si tips, resulting in a pillar with $a \sim 1 \mu \mathrm{m}$ waist diameter.

14. Removal of all oxide
15. Over-oxidization of pillars to form and sharpen tips. The oxide is grown for $5 \mathrm{~h}$ at $1100^{\circ} \mathrm{C}$, yielding $\sim 1.5 \mu \mathrm{m}$. All oxide can be removed ( $\mathrm{HF} 48 \% \sim 3 \mathrm{~min}$ ) and applied again to form sharper tips 16. Growth of $\sim 270 \mathrm{~nm}$ oxide $\left(1.5 \mathrm{~h}\right.$ at $\left.925^{\circ} \mathrm{C}\right)$

17. Lithography: Pattern the insulating oxide

18. BHF $6: 1$ to pattern the insulating oxide

19. Ion implantat ohmic contacts between the ground metal planes and the Si body

Steps 20 and 21 for photodiode tips only:

20. Lithography: Open the oxide at the tip by spinning resist and opening it at the top of the tips using $\mathrm{O}_{2}$ plasma

21. BHF 6:1 to open the oxide at the tip

22. Thermal evaporation of $1000 \AA$ metal (Au or Al)

23. Lithography: Patterning the metal waveguide

24. Metal etch to form the waveguide.

25. Lithography: Opening of the metal at the tip only by applying resist and opening it with $\mathrm{O}_{2}$ plasma at tip

26. Metal etch and oxide etch in case of coax tips

27. PECVD Oxide deposition to protect front side of wafer in release step

27a-c for coaxial tips:

27a. Lithography: Pattern front side oxide to form $\mathrm{XeF}_{2}$ etch pattern

27b. BHF 6:1 oxide etch to form the $\mathrm{XeF}_{2}$ etch holes

$27 \mathrm{c}$. $\mathrm{XeF}_{2}$ etch to form suspended center waveguide (see more detailed explanation following this list)

28. Silicon etch to release back side using anisotropic plasma process

29. Oxide etch to remove protective oxide on front side

30. Al evaporation on backside to enhance reflectance from cantilever

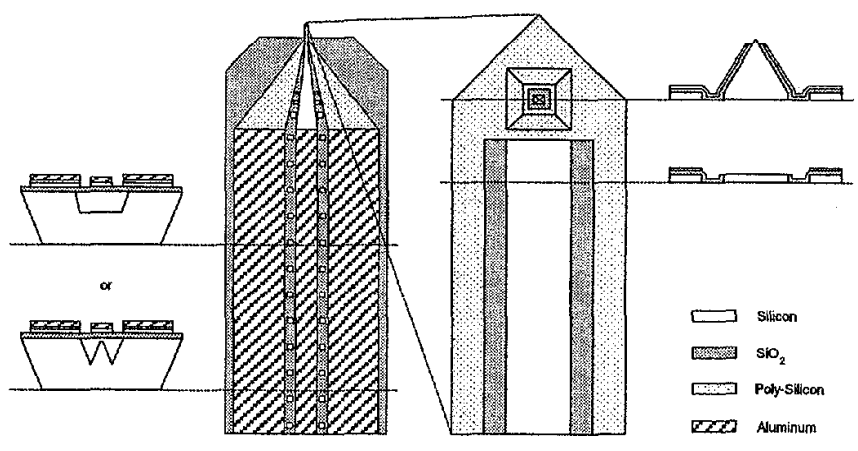

Figure 1. Coaxial SPM tip/cantilever layout. Coplanar waveguide $(C P W)$ transmission line has windows in its gap for $\mathrm{XeF}_{2}$ etching and suspension on oxide membrane.

To undercut the center waveguide, we built and used a small $\mathrm{XeF}_{2}$ etching chamber. It incorporates the chamber, mechanical vacuum pump, etchant reservoir, nitrogen purge line, and computer-controlled valve actuation and pressure acquisition.

A long-working-distance microscope is necessary to observe the etch process since its speed depends on sample geometry, exposed area and etchant pressure.

In order to etch, a single wafer is loaded into the chamber and pressure is reduced to $10 \mathrm{mT}$. Exposing the $\mathrm{XeF}_{2}$ reservoir to the 
same pressure will sublimate some of the $\mathrm{XeF}_{2}$ powder until a pressure of about 2 Torr is reached. This gas is introduced into the etching chamber where it etches any exposed Si surface. For this reason, the backside and rim of the wafer has to be covered with a layer of non-etching tape. The $\mathrm{XeF}_{2}$ is allowed to stay in the etching chamber for about 90-120 seconds after which the chamber is purged and new etchant is introduced. After about 15 cycles, slight warping of the center waveguide can be observed (Figure 2). This is a sign that it has been completely underetched, connected only to the oxide above and the 3-4 suspension posts along the waveguide which are necessary for mechanical stability.

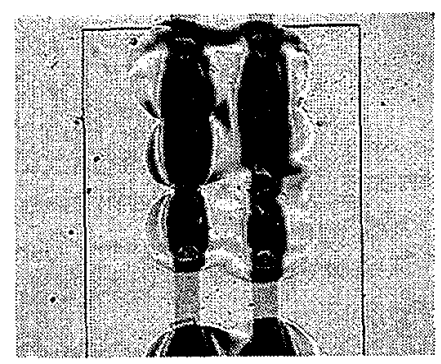

Figure 2. Center waveguide after $\mathrm{XeF}_{2}$ etching.

As an important addition to microfabricating the probe tip, we have designed an interface to quickly exchange scanning tips on the SFM scanner and reconnect its electrical connections without adding height that could hamper SFM operation (Figure 3).

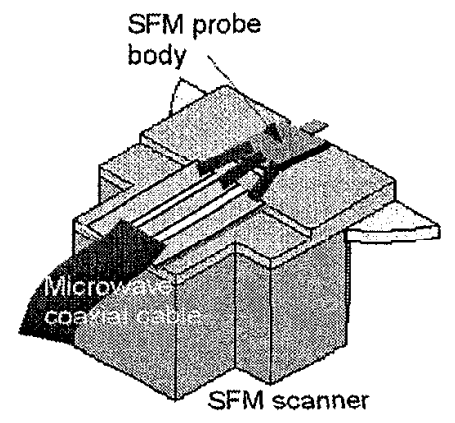

Figure 3. Interface between coaxial tip/SFM body and microwave cable, shown on SFM scanner.

The interface consists of a clamp holder for the SFM chip and scanner, a waveguide transition and a permanently attached microwave coaxial cable. The connection between SFM chip and waveguide transition can be established using gold wire bonding or small clamping springs.

\section{TOPOGRAPHY AND OPTICAL RESULTS}

As shown in the following scans, the coaxial nature of the SFM tip (Fig. 4) does not affect images of typical IC-like structures. Further tests of the probe's optical response showed that it is single-aperture with no parasitic junctions.

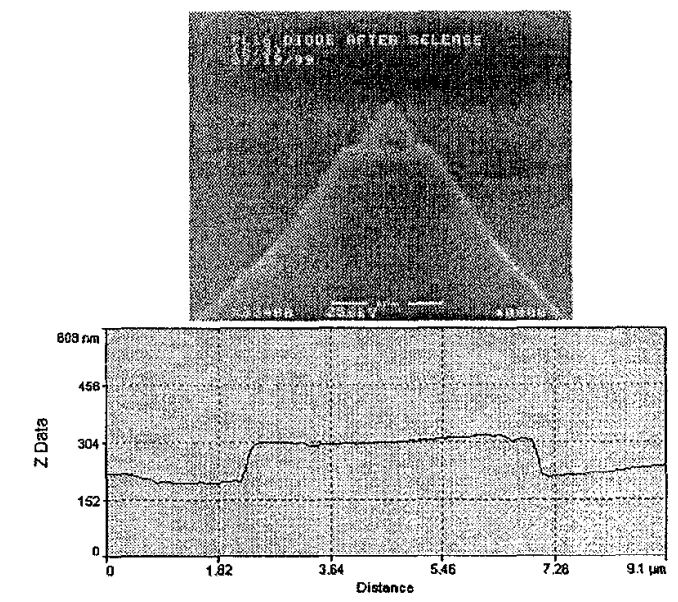

Figure 4. Coaxial tip (top) and line scan using tip (bottom)

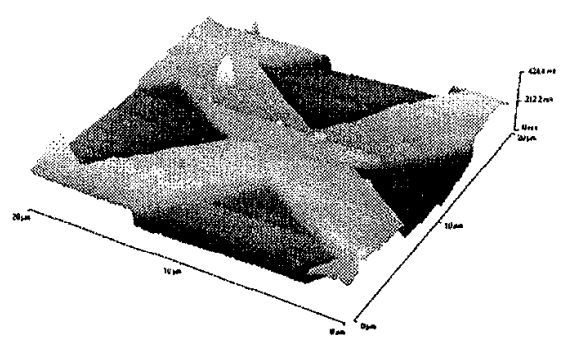

Figure 5. Topographical image of test sample.

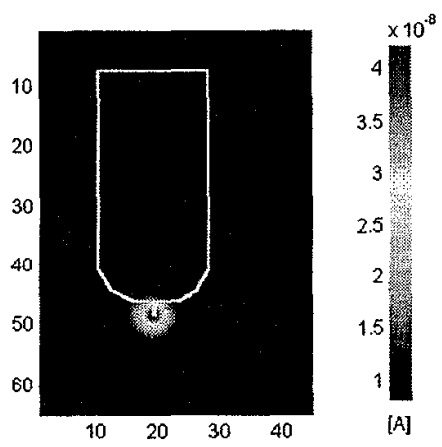

Figure 6. Scan with HeNe laser over photodetector tip and body $(20 \times 30 \mathrm{~mm}$ area $)$.

The process we have discussed enables complete integration of coaxial (and photodiode) probe tips, low-loss microwave transmission lines and SFM cantilevers. These will enable a wide range of near-field microwave and optical investigations.

We acknowledge support from DARPA, ONR (MURI), and NSF (PECASE).

\section{REFERENCES}

[1] D. W, van der Weide and P. Neuzil, "The nanoscilloscope: Combined topography and $\mathrm{AC}$ field probing with a micromachined tip," Journal of Vacuum Science \& Technology B, 14, 4144 (1996). 
[2] D. W. van der Weide, "Localized picosecond resolution with a near-field microwave/scanning-force microscope," Applied Physics Letters, 70, 677 (1997).

[3] D. W. van der Weide, "Microscopes for the sub-visible: scanning the near field in the microwave through infrared," Optics and Photonics News, 9, 40 (1998).

[4] R. C. Davis, C. C. Williams, and P. Neuzil, "Micromachined submicrometer photodiode for scanning probe microscopy," Applied Physics Letters, 66, 2309 (1995).

[5] T. Leinhos, M. Stopka, and E. Oesterschulze, "Micromachined fabrication of Si cantilevers with Schottky diodes integrated in the tip," Appl. Phys. A, 66, 65 (1998).

[6] D. Drews, W. Ehrfeld, M. Lacher, K. Mayr, W. Noell, S. Schmitt, and M. Abraham, "Nanostructured probes for scanning near-field optical microscopy,"

Nanotechnology, 10, 61 (1999).

[7] D. W. van der Weide, V. Agrawal, T. Bork, and P. Neuzil, "Localized circuit probing with a combined Schottky diode/scanning force microscope," Technical Digest of the IEEE Microwave Theory and Techniques Symposium, Baltimore, IEEE (1998), pp. 1341.

[8] U. Dürig, D. Pohl, and H. Rohrer, "Near-field optical scanning microscopy," Journal of Applied Physics, 59, 3318 (1986).

[9] E. Betzig, A. Lewis, A. Harootunian, M. Isaacson, and E. Kratschmer, "Near-field scanning optical microscopy (NSOM)," Biophys. J., 49, 269 (1986).

[10] E. Betzig and J. K. Trautman, "Near-Field Optics: Microscopy, Spectroscopy, and Surface Modification Beyond the Diffraction Limit," Science, 257, 189 (1992).

[11] D. Courjon and C. Bainier, "Near field microscopy and near field optics," Rep. Prog. Phys., 57, 989 (1994).

[12] F. Zenhausern, M. P. O'Boyle, and H. K. Wickramasinghe, "Apertureless near-field optical microscope," Applied Physics Letters, 65, 1623 (1994).

[13] P. A. Crowell, D. K. Young, S. Keller, E. L. Hu, and D. D. Awschalom, "Near-field scanning optical spectroscopy of an InGaN quantum well," Applied Physics Letters, 72, 927 (1998).

[14] K. E. Goodson and M. Asheghi, "Near-field optical thermometry," Microscale Thermophysical Engineering, 1, 225 (1997).

[15] P. G. Haydon, S. Marchese-Ragona, T. A. Basarsky, M. Szulczewski, and M. McCloskey, "Near-field confocal optical spectroscopy (NCOS): Subdiffraction optical resolution for biological systems," Journal Of Microscopy, 182, 208 (1996).

[16] E. A. Ash and G. Nicholls, "Super-resolution aperture scanning microscope," Nature, 237, 510 (1972).

[17] M. Fee, S. Chu, and T. W. Hansch, "Scanning electromagnetic transmission line microscope with subwavelength resolution," Optics Communications, 69, 219 (1989).

[18] E. Tanabe and W. T. Joines, "A nondestructive method for measuring the complex permittivity of dielectric materials at microwave frequencies using an open kansmission line resonator," IEEE Transactions on Instrumentation and Measurement, 25, 222 (1976).
[19] C. P. Vlahacos, R. C. Black, S. M. Anlage, A. Amar, and F. C. Wellstood, "Near-field scanning microwave microscope with $100 \mathrm{mu}$ m resolution," Applied Physics Letters, 69, 3272 (1996).

[20] D. E. Steinhauer, C. P. Vlahacos, S. K. Dutta, B. J. Feenstra, F. C. Wellstood, and S. M. Anlage, "Quantitative imaging of sheet resistance with a scanning near-field microwave microscope," Applied Physics Letters, 72, 861 (1998).

[21] T. Wei, X. D. Xiang, W. G. Wallace-Freedman, and P. G. Schultz, "Scanning tip microwave near-field microscope," Applied Physics Letters, 68, 3506 (1996).

[22] Y. Lu, T. Wei, F. Duewer, Y. Lu, N.-B. Ming, P. G. Schultz, and X. D. Xiang, "Nondestructive imaging of dielectric-contrast profiles and ferroelectric domains with a scanning-tip microwave near-field microscope," Science, 276, 2004 (1997).

[23] F. Keilmann, D. W. van der Weide, T. Eickelkamp, R. Merz, and D. Stockle, "Extreme sub-wavelength resolution with a scanning radio-frequency transmission microscope," Optics Communications, 129, 15 (1996).

[24] V. Agrawal, P. Neuzil, and D. W. van der Weide, "A microfabricated tip for simultaneous acquisition of sample topography and high-frequency magnetic field," Applied Physics Letters, 71, 2343 (1997).

[25] N. H. Tea, V. Milanovic, C. A. Zincke, J. S. Suehle, M. Gaitan, M. E. Zaghloul, and J. Geist, "Hybrid postprocessing etching for CMOS-compatible MEMS," Journal of Microelectromechanical Systems, 6, 363 (1997). 